\title{
Combinatorial properties of the enhanced principal rank characteristic sequence over finite fields
}

https://doi.org/10.1515/spma-2021-0154

Received July 15, 2021; accepted December 15, 2021

Abstract: The enhanced principal rank characteristic sequence (epr-sequence) of a symmetric matrix $B \in$ $\mathbb{F}^{n \times n}$ is defined as $\ell_{1} \ell_{2} \cdots \ell_{n}$, where $\ell_{j} \in\{\mathrm{A}, \mathrm{S}, \mathrm{N}\}$ according to whether all, some but not all, or none of the principal minors of order $j$ of $B$ are nonzero. Building upon the second author's recent classification of the epr-sequences of symmetric matrices over the field $\mathbb{F}=\mathbb{F}_{2}$, we initiate a study of the case $\mathbb{F}=\mathbb{F}_{3}$. Moreover, epr-sequences over finite fields are shown to have connections to Ramsey theory and coding theory.

Keywords: Principal minor; symmetric matrix; finite field; enhanced principal rank characteristic sequence; rank

MSC: 15B33, 15B57, 15A15, 15A03, 94B05

\section{Introduction}

For a given positive integer $n,[n]:=\{1,2, \ldots, n\}$. Let $\mathbb{F}$ be a field, $B \in \mathbb{F}^{n \times n}$ and $\alpha, \beta \subseteq[n]$; then $B$ has order $n ; B[\alpha, \beta]$ denotes the submatrix of $B$ lying in rows indexed by $\alpha$ and columns indexed by $\beta$; $B[\alpha, \alpha]:=B[\alpha]$ and is called a principal submatrix; the determinant of a $k \times k$ principal submatrix of $B$ is a principal minor, and such a minor has order $k$. With $\mathbb{F}_{q}$, where $q$ is a prime power, we denote the finite field with $q$ elements.

For a given symmetric matrix $B \in \mathbb{R}^{n \times n}$ and a fixed $k \in[n]$, Brualdi et al. [2] introduced the principal rank characteristic sequence (pr-sequence) of $B$, in which they recorded the existence of at least one (respectively, the nonexistence of any) nonzero principal minor of order $k$ with a 1 (respectively, 0 ) in position $k$; their definition of the pr-sequence was later extended to other fields by Barrett et al. [1]: Let $\mathbb{F}$ be a field and $B \in \mathbb{F}^{n \times n}$ be symmetric; the principal rank characteristic sequence (pr-sequence) of $B$ is defined as $\operatorname{pr}(B)=r_{0} r_{1} \cdots r_{n}$, where, for $k=1,2, \ldots, n$,

$$
r_{k}= \begin{cases}1 & \text { if } B \text { has a nonzero principal minor of order } k \text {; and } \\ 0 & \text { otherwise; }\end{cases}
$$

while $r_{0}=1$ if and only if $B$ has a 0 diagonal entry. As a simplification of the principal minor assignment problem (which is stated in [6]), Brualdi et al. [2] studied what sequences of 0 s and 1s are attained by symmetric matrices over the real field, $\mathbb{R}$, among other matrices. The study of pr-sequences was extended by Barrett et al. [1] to symmetric matrices over other fields, with their focus largely on fields of characteristic 2. Motivated

Peter J. Dukes: Department of Mathematics and Statistics, University of Victoria, Victoria, BC, V8W 2Y2, Canada, E-mail: dukes@uvic.ca

*Corresponding Author: Xavier Martínez-Rivera: Department of Mathematics and Statistics, University of Victoria, Victoria, BC, V8W 2Y2, Canada, E-mail: martinez.rivera.xavier@gmail.com 
by the pr-sequence, and to cast more light on the existence or nonexistence of a nonzero principal minor of a given order in symmetric (and complex Hermitian) matrices, Butler et al. [4] introduced another sequence:

Definition 1.1. [4, Definition 1.1] Let $\mathbb{F}$ be a field. The enhanced principal rank characteristic sequence of $B \in$ $\mathbb{F}^{n \times n}$ (epr-sequence) is defined as $\operatorname{epr}(B)=\ell_{1} \ell_{2} \cdots \ell_{n}$, where

$$
\ell_{j}= \begin{cases}\text { A } & \text { if all of the principal minors of order } j \text { are nonzero; } \\ \mathrm{S} & \text { if some but not all of the principal minors of order } j \text { are nonzero; } \\ \mathrm{N} & \text { if none of the principal minors of order } j \text { are nonzero (i.e., all are zero). }\end{cases}
$$

Results concerning symmetric matrices over various fields, including constructions of matrices attaining certain epr-sequences, as well as results stating that certain subsequences cannot occur in the epr-sequence of a symmetric matrix, were presented in [4]. In [8], Martínez-Rivera established the following (complete) characterization of the epr-sequences of symmetric matrices over the finite field $\mathbb{F}=\mathbb{F}_{2}$, where, for a given sequence $t_{i_{1}} t_{i_{2}} \cdots t_{i_{k}}$ from $\{\mathrm{A}, \mathrm{N}, \mathrm{S}\}, \overline{t_{i_{1}} t_{i_{2}} \cdots t_{i_{k}}}$ indicates that $t_{i_{1}} t_{i_{2}} \cdots t_{i_{k}}$ is repeated as many times as desired, or omitted entirely (i.e., $\overline{t_{i_{1}} t_{i_{2}} \cdots t_{i_{k}}}$ may be vacuous):

Theorem 1.2. [8, Theorems 3.2, 3.8, 3.11] Let $\sigma=\ell_{1} \ell_{2} \cdots \ell_{n}$ be a sequence from $\{\mathrm{A}, \mathrm{N}, \mathrm{S}\}$. Then there exists $a$ symmetric matrix $B \in \mathbb{F}_{2}^{n \times n}$ with $\operatorname{epr}(B)=\sigma$ if and only if $\sigma$ is of one of the following forms:
1. $A \bar{A}$;
8. ASA $\overline{\mathrm{SAN}}$;
15. $S \bar{S} A$;
2. $A \bar{S} N \bar{N}$;
9. $\mathrm{NA} \overline{\mathrm{NA}}$;
16. $\mathrm{S} \overline{\mathrm{SAA}}$;
3. $\mathrm{ASS} \overline{\mathrm{S}} \mathrm{A}$;
10. NA $\overline{N A N}$;
17. SSTAN;
4. $A S S \bar{S} A A$;
11. $\overline{\mathrm{NS}} \mathrm{N} \overline{\mathrm{N}}$;
18. $\mathrm{SASA} \overline{\mathrm{SA}}$;
5. ASSSTSAN with $n$ even;
12. NSTNNA;
19. SASA $\overline{\mathrm{SA}} \mathrm{A}$;
6. $\mathrm{ASA} \overline{\mathrm{SA}}$;
13. NSTNSNAN;

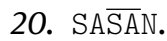
7. ASA $\overline{S A}$;
14. $\mathrm{S} \overline{\mathrm{SN}} \mathrm{N}$;

Here, we build upon Theorem 1.2 by initiating a study of the epr-sequences of symmetric matrices over the field $\mathbb{F}=\mathbb{F}_{3}$, as well as show that the epr-sequences of symmetric matrices over finite fields have connections to Ramsey theory and coding theory. We note that a brief treatment of pr-sequences of symmetric matrices over $\mathbb{F}_{3}$ was presented in [1, p. 231].

At the end of this section, an application of epr-sequences in coding theory, one that is part of our motivation for studying the epr-sequences of symmetric matrices over finite fields, is identified. Section 2 contains preliminary results. In Section 3, a connection between Ramsey theory and the epr-sequences of symmetric matrices over finite fields is established and used to obtain results for epr-sequences over finite fields. Section 4 focuses on the epr-sequences of symmetric matrices over the field $\mathbb{F}=\mathbb{F}_{3}$, with its main result being a complete characterization of those that do not contain any $S$ terms (see Theorem 4.12). Before it concludes with Subsection 1.1, this section introduces some necessary terminology.

For a symmetric matrix $B$ with epr $(B)=\ell_{1} \ell_{2} \cdots \ell_{n}$, $[\operatorname{epr}(B)]_{i}:=\ell_{i}$, for all $i \in[n]$; moreover, for all $i, k \in[n]$ with $i+k \leq n$, $[\operatorname{epr}(B)]_{i}^{i+k}:=\ell_{i} \ell_{i+1} \ell_{i+2} \cdots \ell_{i+k}$. If a given sequence $\ell_{1} \ell_{2} \cdots \ell_{n}$ from $\{\mathrm{A}, \mathrm{N}, \mathrm{S}\}$ is the epr-sequence of a symmetric matrix over a field $\mathbb{F}$, then we shall say that $\ell_{1} \ell_{2} \cdots \ell_{n}$ is an "epr-sequence over $\mathbb{F}$."

Let $a$ and $b$ be positive integers. The Ramsey number $R(a, b)$ is the minimum integer $n$ such that, in any 2-coloring of the edges of the complete graph $K_{n}$, say with colors \{red, blue\}, there is guaranteed to exist either a clique of order $a$, all of whose edges are red, or a clique of order $b$, all of whose edges are blue. The case when $a=b$ is of particular importance; for instance, the well-known Ramsey number $R(3,3)=6$ asserts that any red, blue coloring of $K_{6}$ contains either a red or a blue triangle (while $K_{5}$ has a coloring that avoids this). More generally, the 'multicolor' Ramsey number $R_{m}(a)$ is defined as the minimum integer $n$ such that any $m$-coloring of the edges of $K_{n}$ induces a monochromatic clique of order $a$. The interested reader is invited to read the excellent book [5] for more information on Ramsey numbers, including the fact that they exist for any input parameters. A dynamic survey of bounds on small Ramsey numbers is maintained at [9]. 
For us, all graphs are simple, undirected and loopless. The cycle graph on $n \geq 3$ vertices is denoted by $C_{n}$. Let $\mathbb{F}$ be a field and $G$ be a graph on $n$ vertices; the number of vertices of $G$ is its order; its complement is denoted by $\bar{G} ; G$ is triangle-free if it does contain $C_{3}$ as a subgraph; and $A_{\mathbb{F}}(G) \in \mathbb{F}^{n \times n}$ denotes the adjacency matrix of $G$ over the field $\mathbb{F}$.

For a given matrix $B \in \mathbb{F}^{n \times n}$ (where $\mathbb{F}$ is a field) having a nonsingular principal submatrix $B[\alpha]$, recall that the Schur complement of $B[\alpha]$ in $B$ is the matrix $B / B[\alpha]:=B\left[\alpha^{c}\right]-B\left[\alpha^{c}, \alpha\right](B[\alpha])^{-1} B\left[\alpha, \alpha^{c}\right]$, where $\alpha^{c}=[n] \backslash \alpha$ (see, for example, [10]). Two given matrices $A$ and $B$ are said to be permutationally similar if there exists a permutation matrix $P$ such that $B=P^{\top} A P$; moreover, the block diagonal matrix with the matrices $A$ and $B$ on the diagonal (in that order) is denoted by $A \oplus B$. The $n \times n$ diagonal matrix whose $j$ th diagonal entry is $d_{j}$ is denoted by $\operatorname{diag}\left(d_{1}, d_{2}, \ldots, d_{n}\right)$. With $I_{n}, O_{n}$ and $J_{m, n}$ we denote, respectively, the $n \times n$ identity matrix, the $n \times n$ zero matrix and the $m \times n$ matrix all of whose entries are equal to 1 . For integers $a, b$ and $p$, where $p>0, a \equiv b(\bmod p)$ denotes the congruence modulo $p$ of $a$ and $b$.

\subsection{An application in coding theory}

In this subsection, an application in coding theory of epr-sequences of symmetric matrices over finite fields is identified.

A $q$-ary code is a subset of $\mathbb{F}_{q}^{n}$ for some positive integer $n$, which is called the length of the code. A code $C \subseteq \mathbb{F}_{q}^{n}$ is linear if it is a subspace of $\mathbb{F}_{q}^{n}$; in this case, we have $|C|=q^{k}$, where $k=\operatorname{dim}(C)$. The elements of $C$ are called codewords. A matrix $G \in \mathbb{F}_{q}^{k \times n}$ whose row space equals $C$ is called a generator matrix for the linear code $C$.

The study of coding theory typically aims to control the number of positions in which two elements of $C$ differ. With this in mind, we recall the Hamming distance on $\mathbb{F}_{q}^{n}$ as $d(u, v)=\left|\left\{i: u_{i} \neq v_{i}\right\}\right|$. The minimum distance of a code $C$ is $\min \{d(u, v): u, v \in C, u \neq v\}$. For a linear code $C$, it is easy to see that its minimum distance also equals the least nonzero 'weight' of a codeword; that is, $\min \{w(u): u \in C, u \neq 0\}$, where $w(u)=d(u, 0)$ is the Hamming weight of $u \in \mathbb{F}_{q}^{n}$.

Given a linear code $C \subseteq \mathbb{F}_{q}^{n}$, its dual code is $C^{\perp}=\left\{v \in \mathbb{F}_{q}^{n}: u^{\top} v=0 \forall u \in C\right\}$. A generator matrix $H$ for $C^{\perp}$ is also called a parity-check matrix for $C$. Indeed, $H$ checks for membership in $C$ in the sense that $C=\operatorname{ker}(H)$. The connection with weights is as follows. Given an $m \times n$ matrix with $m<n$, its spark is the least integer $s$ such that there exists a set of $s$ linearly dependent columns in the matrix; this integer $s$ can be viewed as the least weight of a nonzero vector in the kernel of the matrix. It follows that the minimum distance of a linear code $C$ equals the spark of its parity-check matrix $H$.

More generally, the weight enumerator of a linear code $C$ of length $n$ is the polynomial

$$
W(x, y)=\sum_{j=0}^{n} A_{j} x^{j} y^{n-j},
$$

where $A_{j}=|\{u \in C: w(u)=j\}|$, the number of codewords of weight $j$.

The next result connects weights in a linear code to the epr-sequence of a certain symmetric matrix.

Theorem 1.3. Let $C \subseteq \mathbb{F}_{q}^{n}$ be a nonzero linear code, $H$ be a parity-check matrix for $C$ and $\operatorname{epr}\left(H^{\top} H\right)=$ $\ell_{1} \ell_{2} \cdots \ell_{n}$. For all $j \in[n]$, if $C$ has a codeword of weight $j$, then $\ell_{j} \neq$ A. In particular, the minimum distance of $C$ is at least $\min \left\{j \in[n]: \ell_{j} \neq \mathrm{A}\right\}$.

Proof. Let $u \in C$ be a codeword of weight $j>0$ and $u=\left[u_{1}, u_{2}, \ldots, u_{n}\right]^{\top}$, and put $\alpha=\left\{i: u_{i} \neq 0\right\}$ (such a $u$ exists because $C$ is nonzero). Observe that $|\alpha|=j$. As $H u=0$, the columns of $H$ that are indexed by $\alpha$ are linearly dependent. Let us denote the restriction of $H$ to columns in $\alpha$ by $\widetilde{H}$ (i.e., $\widetilde{H}$ is the matrix obtained from $H$ by deleting the columns of $H$ that are not indexed by $\alpha)$. Then, as $\left(H^{\top} H\right)[\alpha]=\widetilde{H}^{\top} \widetilde{H}$ and $\operatorname{rank}\left(\widetilde{H}^{\top} \widetilde{H}\right) \leq \operatorname{rank}(\widetilde{H})<|\alpha|$, the $j \times j$ principal submatrix $\left(H^{\top} H\right)[\alpha]$ is singular, implying that $\ell_{j} \neq \mathrm{A}$.

We remark that, over fields of characteristic zero, the spark of $H$ is exactly $\min \left\{j \in[n]: \ell_{j} \neq A\right\}$, where $\operatorname{epr}\left(H^{\top} H\right)=\ell_{1} \ell_{2} \cdots \ell_{n}$. In this sense, epr-sequences represent a refinement of spark. Over finite fields, the 
converse of Theorem 1.3 can fail, in the sense that $\ell_{j} \neq \mathrm{A}$ in spite of there being no codewords of weight $j$. In particular, it can be shown (using Theorems 2.7 and 4.7) that, for $C \subseteq \mathbb{F}_{q}^{n}$, where $q \in\{2,3\}$, we have $\min \left\{j \in[n]: \ell_{j} \neq \mathrm{A}\right\} \leq q$, independent of the minimum distance of $C$. It may be of interest to explore how close this measure is to the minimum distance for larger $q$.

\section{Preliminary results}

This section contains preliminary results that are needed in subsequent sections, and it starts with some known facts.

\subsection{Results cited}

The following well-known fact (see, for example, [1]) states that the rank of a symmetric matrix $B$ is equal to the order of a largest nonsingular principal submatrix of $B$; because of this, we shall call the rank of a symmetric matrix "principal."

Theorem 2.1. [1, Theorem 1.1] Let $\mathbb{F}$ be a field and $B \in \mathbb{F}^{n \times n}$ be symmetric. Then $\operatorname{rank}(B)=\max \{|\gamma|$ : $\operatorname{det}(B[\gamma]) \neq 0\}$, where the maximum over the empty set is defined to be 0 .

If $B$ is a given nonsingular symmetric matrix, then the epr-sequence of $B^{-1}$ is obtained readily from that of $B$ :

Theorem 2.2. [4, Theorem 2.4] (Inverse Theorem.) Let $\mathbb{F}$ be a field and $B \in \mathbb{F}^{n \times n}$ be symmetric. Suppose that $B$ is nonsingular. If $\operatorname{epr}(B)=\ell_{1} \ell_{2} \cdots \ell_{n-1} \mathrm{~A}$, then $\operatorname{epr}\left(B^{-1}\right)=\ell_{n-1} \ell_{n-2} \cdots \ell_{1} \mathrm{~A}$.

If $B$ is a given matrix and $C$ is a principal submatrix of $B$, then some of the terms in epr $(C)$ may be deduced from those in $\operatorname{epr}(B)$, assuming that the latter is known:

Theorem 2.3. [4, Theorem 2.6] (Inheritance Theorem.) Let $\mathbb{F}$ be a field, $B \in \mathbb{F}^{n \times n}$ be symmetric, $m \leq n$ and $1 \leq i \leq m$. Then the following statements hold:

1. If $[\operatorname{epr}(B)]_{i}=\mathrm{N}$, then $[\operatorname{epr}(C)]_{i}=\mathrm{N}$, for all $m \times m$ principal submatrices $C$.

2. If $[\operatorname{epr}(B)]_{i}=\mathrm{A}$, then $[\operatorname{epr}(C)]_{i}=\mathrm{A}$, for all $m \times m$ principal submatrices $C$.

3. If $[\operatorname{epr}(B)]_{m}=\mathrm{S}$, then there exist $m \times m$ principal submatrices $C_{A}$ and $C_{N}$ of $B$ such that $\left[\operatorname{epr}\left(C_{A}\right)\right]_{m}=\mathrm{A}$ and $\left[\operatorname{epr}\left(C_{N}\right)\right]_{m}=\mathrm{N}$.

4. If $i<m$ and $[\operatorname{epr}(B)]_{i}=\mathrm{S}$, then there exists an $m \times m$ principal submatrix $C_{S}$ such that $\left[\operatorname{epr}\left(C_{S}\right)\right]_{i}=\mathrm{S}$.

We shall need the following well-known fact about Schur complements.

Theorem 2.4. (Schur Complement Theorem.) Let $\mathbb{F}$ be a field, $B \in \mathbb{F}^{n \times n}$ be symmetric, $\alpha \subset[n], \alpha^{c}=[n] \backslash \alpha$ and $|\alpha|=k$. Suppose that $B[\alpha]$ is nonsingular, and let $S=B / B[\alpha]$. Then the following statements hold:

(i) $S$ is an $(n-k) \times(n-k)$ symmetric matrix.

(ii) [3, p. 771] If the indexing of $S$ is inherited from $B$, then, for all $\gamma \subseteq \alpha^{c}$,

$$
\operatorname{det}(S[\gamma])=\frac{\operatorname{det}(B[\gamma \cup \alpha])}{\operatorname{det}(B[\alpha])} .
$$

If there are two consecutive Ns in the epr-sequence of a symmetric matrix, then each letter in the sequence from that point forward is $\mathrm{N}$ : 
Theorem 2.5. [4, Theorem 2.3] (NN Theorem.) Let $\mathbb{F}$ be a field, $B \in \mathbb{F}^{n \times n}$ be symmetric and epr $(B)=\ell_{1} \ell_{2} \cdots \ell_{n}$. Suppose that $\ell_{k}=\ell_{k+1}=\mathrm{N}$, for some $k$. Then, for all $j \geq k, \ell_{j}=\mathrm{N}$.

The epr-sequence of any symmetric matrix over a field of characteristic not 2 has neither NAN nor NAS as a subsequence:

Theorem 2.6. [4, Theorem 2.14] Let $\mathbb{F}$ be a field of characteristic not 2, and let $B \in \mathbb{F}^{n \times n}$ be symmetric. Then neither NAN nor NAS are subsequences of $\operatorname{epr}(B)$.

We shall reference Theorem 2.6 by saying that "NAN is forbidden" (likewise with NAS).

The next theorem is concerned with symmetric matrices over the field $\mathbb{F}=\mathbb{F}_{2}$.

Theorem 2.7. [8, Theorem 2.9] Let $B \in \mathbb{F}_{2}^{n \times n}$ be symmetric and $\operatorname{epr}(B)=\ell_{1} \ell_{2} \cdots \ell_{n}$. Suppose that AA is a subsequence of $\ell_{1} \ell_{2} \cdots \ell_{n-1}$. Then $\operatorname{epr}(B)=\bar{A} A A A \bar{A}$.

The following fact is well-known (see, for example, [5]).

Theorem 2.8. Let $G$ be a graph of order 5 . If $G$ and $\bar{G}$ are triangle-free, then $G=C_{5}$.

\subsection{Other preliminary results}

The fact that the rank of any matrix remains invariant after being multiplied by a nonzero constant, or after permuting one of its rows or columns, leads to an observation.

Observation 2.9. Let $\mathbb{F}$ be a field and $B \in \mathbb{F}^{n \times n}$ be symmetric. Let $c \in \mathbb{F}$ be nonzero and $P \in \mathbb{F}^{n \times n}$ be $a$ permutation matrix. Then $c B$ and $P^{\top} B P$ are symmetric, $\operatorname{epr}(c B)=\operatorname{epr}(B)$ and $\operatorname{epr}\left(P^{\top} B P\right)=\operatorname{epr}(B)$.

Observation 2.10. Let $\mathbb{F}$ be a field and $B=\left[b_{i j}\right] \in \mathbb{F}^{n \times n}$ be symmetric. Let $D=\operatorname{diag}\left(d_{1}, d_{2}, \ldots, d_{n}\right) \in \mathbb{F}^{n \times n}$ be nonsingular, $M=D B D$ and $M=\left[m_{i j}\right]$. Then the following statements hold:

(i) $m_{i j}=d_{i} d_{j} b_{i j}$, for all $i, j \in[n]$. In particular, if $b_{1 j} \neq 0$ for $j=2,3, \ldots, n$ and $D=\operatorname{diag}\left(1, b_{12}^{-1}, b_{13}^{-1}, \ldots, b_{1 n}^{-1}\right)$, then each off-diagonal entry in the first row (and first column) of $M$ is equal to 1.

(ii) $M$ is symmetric.

(iii) $\operatorname{epr}(M)=\operatorname{epr}(B)$.

Statement (i) in Observation 2.10 is readily verified; statement (ii) follows from (i); and statement (iii) is a consequence of the fact that multiplying any row or column of a matrix by a nonzero constant preserves the rank of each of its submatrices.

The following fact generalizes [4, Theorem 5.1] and, to some extent, [2, Theorem 8.1], which provided the idea for our proof.

Proposition 2.11. Let $\mathbb{F}$ be a field and $B \in \mathbb{F}^{n \times n}$ be symmetric. Suppose that $\operatorname{epr}(B)=\mathrm{AN}_{3} \ell_{4} \cdots \ell_{n}$. Then there exists a symmetric matrix $M \in \mathbb{F}^{n \times n}$ such that the following statements hold:

1. $\operatorname{epr}(M)=\operatorname{epr}(B)$

2. Each entry of $M$ is equal to either -1 or 1 .

3. Each diagonal entry of $M$ and each entry in the first row and first column of $M$ is equal to 1.

Proof. It is readily verified that the assumption that $[\operatorname{epr}(B)]_{1}^{2}=$ AN implies that each entry of $B$ is nonzero. Let $B=\left[b_{i j}\right], C=b_{11}^{-1} B$ and $C=\left[c_{i j}\right]$. It follows, then, that each entry of $C$ is nonzero; in particular, observe 
that $c_{11}=1$. Let $D=\operatorname{diag}\left(c_{11}^{-1}, c_{12}^{-1}, \ldots, c_{1 n}^{-1}\right), M=D C D$ and $M=\left[m_{i j}\right]$. Observe that $M$ is symmetric. By Observations 2.9 and 2.10, $\operatorname{epr}(M)=\operatorname{epr}(C)=\operatorname{epr}(B)$.

By Observation 2.10, $m_{11}=c_{11}^{-1}=1$ and each off-diagonal entry in the first row and first column of $M$ is 1 , as desired. As $[\operatorname{epr}(M)]_{2}=\mathrm{N}, 0=\operatorname{det}(M[\{1, j\}])=m_{j j}-1$, for $j=2,3, \ldots, n$. Thus, each diagonal entry of $M$ is 1 , as desired.

Then, as $[\operatorname{epr}(M)]_{2}=\mathrm{N}, 0=\operatorname{det}(M[\{i, j\}])=1-m_{i j}^{2}$, for all $i, j \in[n]$ with $i \neq j$. Hence, each off-diagonal entry of $M$ is either -1 or 1 .

Proposition 2.11 implies that to find an $n \times n$ symmetric matrix (over a given field $\mathbb{F}$ ) whose epr-sequence is of the form $\mathrm{AN}_{3} \ell_{4} \cdots \ell_{n}$ (if it exists), it suffices to search among the subset of symmetric matrices in $\mathbb{F}^{n \times n}$ all of whose entries are either -1 or 1 and whose entries in the first row (and first column) and the diagonal are all equal to 1.

We draw upon the ideas of [2, Theorem 2.2] to make the following observation.

Observation 2.12. Let $n \geq 1$ be an integer, $\mathbb{F}$ be a field and $k \in \mathbb{F}$ be nonzero. Over the field $\mathbb{F}$, $\operatorname{det}\left(U_{n}-k I_{n}\right)=$ $(-k)^{n-1}(n-k)$.

We note that, as each $i \times i$ principal submatrix of $J_{n}-k I_{n}$ (for a given $i \in[n]$ ) is of the form $J_{i}-k I_{i}$, all of the order-i principal minors of $J_{n}-k I_{n}$ are equal, implying that the epr-sequence of $J_{n}-k I_{n}$ does not have any $\mathrm{S}$ terms. Thus, the next two facts follow from Observation 2.12 (the first of which generalizes [4, Proposition 2.17]).

Proposition 2.13. Let $\mathbb{F}$ be a field of characteristic $p>0, J_{n}-I_{n} \in \mathbb{F}^{n \times n}$ and $\operatorname{epr}\left(J_{n}-I_{n}\right)=\ell_{1} \ell_{2} \cdots \ell_{n}$. Then, for all $i \in[n], \ell_{i} \in\{\mathrm{A}, \mathrm{N}\}$. Moreover, for all $i \in[n], \ell_{i}=\mathrm{N}$ if and only if $i \equiv 1(\bmod p)$.

Proposition 2.14. Let $\mathbb{F}$ be a field of characteristic $p>2, J_{n}-2 I_{n} \in \mathbb{F}^{n \times n}$ and $\operatorname{epr}\left(J_{n}-2 I_{n}\right)=\ell_{1} \ell_{2} \cdots \ell_{n}$. Then, for all $i \in[n], \ell_{i} \in\{\mathrm{A}, \mathrm{N}\}$. Moreover, for all $i \in[n], \ell_{i}=\mathrm{N}$ if and only if $i \equiv 2(\bmod p)$.

We draw upon the ideas of [7, Proposition 2.5] to establish the next fact.

Proposition 2.15. Let $\mathbb{F}$ be a field of characteristic $p>2$ and $B \in \mathbb{F}^{n \times n}$ be symmetric. Suppose that epr $(B)=$ $\mathrm{ANA}_{4} \ell_{5} \cdots \ell_{n}$. Then, for $i=4,5, \ldots, n, \ell_{i} \in\{\mathrm{A}, \mathrm{N}\}$. Moreover, for $i=4,5, \ldots, n, \ell_{i}=\mathrm{N}$ if and only if $i \equiv 2$ $(\bmod p)$.

Proof. Because of Proposition 2.14, it suffices to show that $\operatorname{epr}(B)=\operatorname{epr}\left(J_{n}-2 I_{n}\right)$. Because of Proposition 2.11, we may assume, without loss of generality, that each entry of $B$ is equal to either -1 or 1 , and that each entry in the first row (and first column) and the diagonal of $B$ is equal to 1 . We now show that

$$
B=\left(\begin{array}{c|c}
1 & J_{1, n-1} \\
\hline J_{n-1,1} & 2 I_{n-1}-J_{n-1}
\end{array}\right),
$$

by showing that each off-diagonal entry of the $(n-1) \times(n-1)$ submatrix $B[\{2,3, \ldots, n\}]$ is equal to -1 . Let $i, j \in\{2,3, \ldots, n\}$ with $i \neq j$. Observe that $\operatorname{det}(B[\{1, i, j\}])=-\left(b_{i j}-1\right)^{2}$. Then, as $[\operatorname{epr}(B)]_{3}=\mathrm{A}$, and because $b_{i j} \in\{-1,1\}, b_{i j}=-1$. Thus, (1) holds. Let $M$ be the (symmetric) matrix that results from multiplying both the first row and first column of $-B$ by -1 . As multiplying a row or a column of a matrix by a nonzero constant leaves the rank of each of its submatrices invariant, $\operatorname{epr}(M)=\operatorname{epr}(-B)$. Observe that $M=J_{n}-2 I_{n}$. Then, as $\operatorname{epr}(-B)=\operatorname{epr}(B)$ (see Observation 2.9), $\operatorname{epr}(B)=\operatorname{epr}(M)=\operatorname{epr}\left(J_{n}-2 I_{n}\right)$. 


\section{Ramsey theory}

In this section, a connection between Ramsey theory and epr-sequences of symmetric matrices over finite fields is established and used to obtain results for epr-sequences over finite fields. The key idea is to interpret off-diagonal entries as edge colors. In this way, Ramsey numbers give information on epr-sequences of sufficiently large matrices over finite fields.

We shall exploit the following observation in this section.

Observation 3.1. Let $\mathbb{F}$ be a field, $B \in \mathbb{F}^{n \times n}$ be symmetric and $B=\left[b_{i j}\right]$. Let $T \subseteq \mathbb{F}$ be finite and nonempty. Suppose that, for all $i, j \in[n], b_{i j} \in T$ if $i \neq j$. If $n \geq R_{|T|}(k)$, then there exists $c \in T$ such that $B$ has a $k \times k$ principal submatrix all of whose off-diagonal entries are equal to $c$.

We start by focusing on epr-sequences whose first term is $\mathrm{N}$. Observe that, for a given matrix $B$ with $\operatorname{epr}(B)=$ $\ell_{1} \ell_{2} \cdots \ell_{n}$ and $\operatorname{pr}(B)=r_{0} r_{1} \cdots r_{n}, \ell_{1}=\mathbb{N}$ if and only if $r_{0} r_{1}=10$; and that, for $j=2,3, \ldots, n, \ell_{j}=\mathrm{N}$ if and only if $r_{j}=0$. In the theorem that we shall now state, which was established in [1], for a given sequence $t_{i_{1}} t_{i_{2}} \cdots t_{i_{k}}$ from $\{0,1\}, \overline{t_{i_{1}} t_{i_{2}} \cdots t_{i_{k}}}$ indicates that $t_{i_{1}} t_{i_{2}} \cdots t_{i_{k}}$ is repeated as many times as desired, or omitted entirely (i.e., $\overline{t_{i_{1}} t_{i_{2}} \cdots t_{i_{k}}}$ may be vacuous); moreover, this theorem establishes a complete characterization of the pr-sequences of symmetric matrices over fields of characteristic 2 :

Theorem 3.2. [1, Theorem 3.1] Let $n \geq 2, \mathbb{F}$ be a field of characteristic 2 and $\sigma=r_{0} r_{1} \cdots r_{n}$ be a sequence from $\{0,1\}$. Then there exists a symmetric matrix in $\mathbb{F}^{n \times n}$ whose pr-sequence is $\sigma$ if and only if $\sigma$ is of one of the following forms:

$$
01 \overline{1} \overline{0}, \quad 1 \overline{01} \overline{0}, \quad 11 \overline{1} \overline{0} \text {. }
$$

The following fact follows immediately from Theorem 3.2:

Theorem 3.3. Let $\mathbb{F}$ be a field of characteristic 2 and $B \in \mathbb{F}^{n \times n}$ be symmetric with $\operatorname{epr}(B)=\mathrm{N}_{2} \ell_{3} \cdots \ell_{n}$. If $j \in[n]$ is odd, then $\ell_{j}=\mathrm{N}$.

With $p:=2$ (the characteristic of $\mathbb{F}$ in Theorem 3.3), the conclusion of Theorem 3.3 may be stated as follows: If $j \in[n]$ and $j \equiv 1(\bmod p)$, then $\ell_{j}=\mathbb{N}$; something that is somewhat reminiscent of that can be said for finite fields: Our next theorem leads to the following assertion: If $B \in \mathbb{F}_{q}^{n \times n}$, where $p$ is a prime and $q$ is a power of $p$, is a symmetric matrix whose epr-sequence starts with $\mathrm{N}$, then there exists an integer $m$ such that the following statement holds if $n \geq R_{q}(m)$ : If $j \in[m]$ and $j \equiv 1(\bmod p)$, then $\ell_{j} \in\{\mathrm{N}, \mathrm{S}\}$.

Theorem 3.4. Let $p$ be a prime, $q$ be a power of $p$ and $B \in \mathbb{F}_{q}^{n \times n}$ be symmetric with $\operatorname{epr}(B)=\mathrm{N}_{2} \ell_{3} \cdots \ell_{n}$. Suppose that $n \geq R_{q}(k p+1)$, for some positive integer $k$. Then, for $i=1,2, \ldots, k, \ell_{i p+1} \in\{\mathrm{N}, \mathrm{S}\}$.

Proof. Let $i \in[k]$. As $n \geq R_{q}(k p+1)$, and because each diagonal entry of $B$ is 0 , Observation 3.1 implies that there exists $c \in \mathbb{F}_{3}$ such that $B$ has a $(k p+1) \times(k p+1)$ principal submatrix all of whose off-diagonal entries are equal to $c$; as each diagonal entry of $B$ is 0 , this submatrix is $c\left(J_{k p+1}-I_{k p+1}\right)$. Let $\alpha \subseteq[n]$ be such that $B[\alpha]=c\left(J_{k p+1}-I_{k p+1}\right)$. Observe that each $(i p+1) \times(i p+1)$ principal submatrix of $B[\alpha]$ is of the form $c\left(J_{i p+1}-I_{i p+1}\right)$, whose determinant is $c^{i p+1}(-1)^{i p}(i p)=0$ (see Observation 2.12). Then, as each principal submatrix of $B[\alpha]$ is also a principal submatrix of $B, \ell_{i p+1} \neq \mathrm{A}$.

The following is a corollary to Theorem 3.4, and it follows from the fact that $R_{3}(4)=R(4,4,4) \leq 230$ (see, for example, [9]).

Corollary 3.5. Let $B \in \mathbb{F}_{3}^{n \times n}$ be symmetric with $\operatorname{epr}(B)=\mathrm{N} \ell_{2} \ell_{3} \cdots \ell_{n}$. Suppose that $n \geq 230$. Then $\ell_{4} \in\{\mathrm{N}, \mathrm{S}\}$.

We shall now consider the case in which the epr-sequence of the (symmetric) matrix $B$ in Theorem 3.4 starts with NA; observe that, in that case, $B$ is not only a matrix all of whose diagonal entries are zero but also a 
matrix all of whose off-diagonal entries are nonzero. We start by making an observation related to the third term in the epr-sequence of such a matrix. If $B=\left[b_{i j}\right]$ is a symmetric matrix whose epr-sequence starts with NA, then $\operatorname{det}(B[\{p, q, r\}])=2 b_{p q} b_{p r} b_{q r}$ (if $\left.p<q<r\right)$. The following observation, then, follows immediately.

Observation 3.6. Let $B \in \mathbb{F}_{q}^{n \times n}$ be symmetric with $\operatorname{epr}(B)=\mathrm{NA}_{3} \ell_{4} \cdots \ell_{n}$. Then the following statements hold:

1. If $\mathbb{F}_{q}$ is of characteristic 2, then $\ell_{3}=\mathrm{N}$.

2. If $\mathbb{F}_{q}$ is not of characteristic 2 , then $\ell_{3}=\mathrm{A}$.

The next theorem improves upon Theorem 3.4 in the case where epr(B) starts with NA.

Theorem 3.7. Let $p$ be a prime, $q$ be a power of $p$ and $B \in \mathbb{F}_{q}^{n \times n}$ be symmetric with epr $(B)=\mathrm{NA}_{3} \ell_{4} \cdots \ell_{n}$. Suppose that $n \geq R_{q-1}(k)+1$, for some positive integer $k$. Then, for $i=1,2, \ldots, k+1$, the following statements hold:

(i) If $i \equiv 1(\bmod p)$, then $\ell_{i} \in\{\mathrm{N}, \mathrm{S}\}$.

(ii) If $i \not \equiv 1(\bmod p)$, then $\ell_{i} \in\{\mathrm{A}, \mathrm{S}\}$.

Proof. Let $i \in[k+1]$. As $[\operatorname{epr}(B)]_{1}^{2}=\mathrm{NA}$, each off-diagonal entry of $B$ is nonzero. Because of Observation 2.10, we may assume, without loss of generality, that each entry in the first row and first column of $B$ is equal to 1 . As $n-1 \geq R_{q-1}(k)$, Observation 3.1 implies that there exists a nonzero element $c \in \mathbb{F}_{q}$ such that the $(n-1) \times(n-1)$ principal submatrix $B[\{2,3, \ldots, n\}]$ has a $k \times k$ principal submatrix all of whose off-diagonal entries are equal to $c$; as each diagonal entry of $B$ is 0 , this $k \times k$ submatrix is $c\left(J_{k}-I_{k}\right)$. Let $\alpha \subseteq\{2,3, \ldots, n\}$ be such that $B[\alpha]=c\left(J_{k}-I_{k}\right)$. Let $M$ be the matrix that results from multiplying the first row and first column of $B$ by $c$. It follows, then, that $M[\{1\} \cup \alpha]=c\left(J_{k+1}-I_{k+1}\right)$. Observe that each $i \times i$ principal submatrix of $M[\{1\} \cup \alpha]$ is of the form $c\left(J_{i}-I_{i}\right)$, whose determinant is $\operatorname{det}\left(c\left(J_{i}-I_{i}\right)\right)=c^{i} \operatorname{det}\left(J_{i}-I_{i}\right)=c^{i}(-1)^{i-1}(i-1)$ (by Observation 2.12). Then, as each principal submatrix of $M[\{1\} \cup \alpha]$ is also a principal submatrix of $M$, it follows that $\ell_{i} \neq \mathrm{A}$ if $i \equiv 1(\bmod p)$, and that $\ell_{i} \neq \mathrm{N}$ if $i \not 1(\bmod p)$.

We shall rely on Observation 3.6 rather than Theorem 3.7 to make assertions about the third term in the eprsequence of a symmetric matrix, as the former leads to stronger assertions.

The following is a corollary to Theorem 3.7, and it follows from the well-known fact that $R_{2}(3)=R(3,3)=$ 6, $R_{2}(4)=R(4,4)=18$ and $R_{2}(5)=R(5,5) \leq 48$ (see, for example, [9]).

Corollary 3.8. Let $B \in \mathbb{F}_{3}^{n \times n}$ be symmetric with $\operatorname{epr}(B)=\mathrm{NA}_{3} \ell_{4} \cdots \ell_{n}$. Then the following statements hold:

(i) If $n \geq 7$, then $\ell_{4} \in\{\mathrm{N}, \mathrm{S}\}$; and

(ii) if $n \geq 19$, then $\ell_{5} \in\{\mathrm{A}, \mathrm{S}\}$; and

(iii) if $n \geq 49$, then $\ell_{6} \in\{A, S\}$.

The following example shows that the first assertion in Corollary 3.8 does not hold for $n<7$, and that the second assertion does not hold for $n<19$.

Example 3.9. For the following $6 \times 6$ symmetric matrix over $\mathbb{F}_{3}$, it is readily verified that $\operatorname{epr}\left(M_{\sigma}\right)=\sigma$ :

$$
M_{\text {NAAANA }}=\left(\begin{array}{c|ccccc}
0 & 1 & 1 & 1 & 1 & 1 \\
\hline 1 & 0 & 1 & 2 & 2 & 1 \\
1 & 1 & 0 & 1 & 2 & 2 \\
1 & 2 & 1 & 0 & 1 & 2 \\
1 & 2 & 2 & 1 & 0 & 1 \\
1 & 1 & 2 & 2 & 1 & 0
\end{array}\right) .
$$


Observe that for fields of characteristic 2, the assertions that one can make based on Theorem 3.7 are not as strong as those based on Theorem 3.3. Thus, we shall not state any corollaries to Theorem 3.7 in the case where $q=4$ or any other power of 2 .

The next fact is another corollary to Theorem 3.7, and it follows from the well-known fact that $R_{4}(3)=$ $R(3,3,3,3) \leq 64$ (see, for example, [9]).

Corollary 3.10. Let $B \in \mathbb{F}_{5}^{n \times n}$ be symmetric with epr $(B)=N \mathrm{NA}_{3} \ell_{4} \cdots \ell_{n}$. Suppose that $n \geq 65$. Then $\ell_{4} \in\{\mathrm{A}, \mathrm{S}\}$.

We close this section by applying Observation 3.1 to make an assertion about epr-sequences that start with AN.

Theorem 3.11. Let $B \in \mathbb{F}_{3}^{n \times n}$ be symmetric with $\operatorname{epr}(B)=\mathrm{AN}_{3} \ell_{4} \cdots \ell_{n}$. Suppose that $n \geq R(3 k+1,3 k+1)+1$, for some positive integer $k$. Then, for $i=1,2, \ldots, k, \ell_{3 i+2} \in\{\mathrm{N}, \mathrm{S}\}$.

Proof. Let $i \in[k]$. Because of Proposition 2.11, we may assume that each entry of $B$ is equal to either -1 or 1 , and that each entry on the diagonal, the first row and the first column of $B$ is equal to 1 . As $n-1 \geq$ $R(3 k+1,3 k+1)$, Observation 3.1 implies that there exists a nonzero element $c \in \mathbb{F}_{3}$ such that $B[\{2,3, \ldots n\}]$ (whose order is $n-1)$ has a $(3 k+1) \times(3 k+1)$ principal submatrix all of whose off-diagonal entries are equal to $c$; as each diagonal entry of $B$ is equal to 1 , this submatrix is $c\left(J_{3 k+1}-I_{3 k+1}\right)+I_{3 k+1}$. Let $\alpha \subseteq\{2,3, \ldots, n\}$ be such that $B[\alpha]=c\left(J_{3 k+1}-I_{3 k+1}\right)+I_{3 k+1}$. As $c^{2}=1$ (because the square of any nonzero element in $\mathbb{F}_{3}$ is equal to 1$), B[\alpha]=c\left(J_{3 k+1}-(1-c) I_{3 k+1}\right)$. Let $M$ be the matrix that results from multiplying the first row and first column of $B$ by $c$. As multiplying a row or a column of a matrix by a nonzero constant leaves the rank of each of its submatrices invariant, $\operatorname{epr}(M)=\operatorname{epr}(B)$. Thus, it suffices to establish the desired conclusion for $M$. Observe that each entry in the first row and first column of $M$ is equal to $c$, and that the $(1,1)$-entry of $M$ is $c^{2}=1$. Thus, $M[\{1\} \cup \alpha]=c\left(J_{3 k+2}-(1-c) I_{3 k+2}\right)$. Observe that each $(3 i+2) \times(3 i+2)$ principal submatrix of $M[\{1\} \cup \alpha]$ is of the form $c\left(J_{3 i+2}-(1-c) I_{3 i+2}\right)$. By Observation 2.12,

$$
\begin{aligned}
\operatorname{det}\left(c\left(J_{3 i+2}-(1-c) I_{3 i+2}\right)\right) & =c^{3 i+2} \operatorname{det}\left(J_{3 i+2}-(1-c) I_{3 i+2}\right) \\
& =c^{3 i+2}(-(1-c))^{3 i+1}(3 i+2-(1-c)) \\
& =c^{3 i+2}(c-1)^{3 i+1}(3 i+c+1) .
\end{aligned}
$$

As $c$ is nonzero, either $c=1$ or $c=2$. Thus, $\operatorname{det}\left(c\left(J_{3 i+2}-(1-c) I_{3 i+2}\right)\right)=0$. Then, as each principal submatrix of $M[\{1\} \cup \alpha]$ is also a principal submatrix of $M, \ell_{3 i+2} \neq \mathrm{A}$.

Applying Theorem 3.11 and the fact that $R(4,4)=18$ (see, for example, [9]), leads to the following corollary.

Corollary 3.12. Let $B \in \mathbb{F}_{3}^{n \times n}$ be symmetric with $\operatorname{epr}(B)=\mathrm{AN}_{3} \ell_{4} \cdots \ell_{n}$. Suppose that $n \geq 19$. Then $\ell_{5} \in\{\mathrm{N}, \mathrm{S}\}$.

\section{Symmetric matrices over $\mathbb{F}_{3}$}

This section focuses on the epr-sequences of symmetric matrices over the field $\mathbb{F}=\mathbb{F}_{3}$, with its main result being a complete characterization of those that do not contain any $\mathrm{S}$ terms (see Theorem 4.12).

Before restricting our focus to the field $\mathbb{F}_{3}$, we state two facts relating to not only $\mathbb{F}_{3}$ but also all fields of characteristic 3, the first of which follows immediately from Propositions 2.13 and 2.14: 
Proposition 4.1. The following statements hold over a field of characteristic 3:

1. If $n \equiv 1(\bmod 3)$, then $\operatorname{epr}\left(J_{n}-I_{n}\right)=\overline{\mathrm{NAAN}}$ and $\operatorname{epr}\left(J_{n}-2 I_{n}\right)=\overline{\text { ANAA. }}$.

2. If $n \equiv 2(\bmod 3)$, then $\operatorname{epr}\left(J_{n}-I_{n}\right)=\overline{\mathrm{NAANA}}$ and $\operatorname{epr}\left(J_{n}-2 I_{n}\right)=\overline{\mathrm{ANAAN}}$.

3. If $n \equiv 3(\bmod 3)$, then $\operatorname{epr}\left(U_{n}-I_{n}\right)=\overline{\text { NAANAA }}$ and epr $\left(J_{n}-2 I_{n}\right)=\overline{\text { ANAANA. }}$

The following statement follows immediately from Proposition 2.15.

Proposition 4.2. Let $\mathbb{F}$ be a field of characteristic 3 and $B \in \mathbb{F}^{n \times n}$ be symmetric. Suppose that epr $(B)=$ $\mathrm{ANA}_{4} \ell_{5} \cdots \ell_{n}$. Then $\operatorname{epr}(B)$ is of one of the following forms:

$$
\text { ANA } \overline{A N A}, \quad \text { ANA } \overline{A N A} A, \quad \text { ANA } \overline{A N A} A N \text {. }
$$

The next fact will be used throughout these pages without being referenced.

Observation 4.3. If $a \in \mathbb{F}_{3}$ is nonzero, then $a^{2}=1$.

An epr-sequence over $\mathbb{F}_{3}$ that starts with AA does not have, roughly speaking, any N terms in the first half of the sequence.

Proposition 4.4. Let $B \in \mathbb{F}_{3}^{n \times n}$ be symmetric. Suppose that $\operatorname{epr}(B)=\mathrm{AA}_{3} \ell_{4} \cdots \ell_{n}$. Then, for all $3 \leq j \leq\left\lceil\frac{n}{2}\right\rceil$, $\ell_{j} \neq N$.

Proof. As multiplying a matrix by a nonzero constant leaves its epr-sequence invariant (see Observation 2.9), and because the square of any nonzero number in $\mathbb{F}_{3}$ is 1 , we may assume, without loss of generality, that the number of diagonal entries of $B$ that are equal to 1 is larger than the number of diagonal entries that are equal to 2. Let $p$ be the number of diagonal entries of $B$ that are equal to $1\left(\operatorname{as}[\operatorname{epr}(B)]_{1}=A, p \neq 0\right)$. As any two permutationally similar matrices have the same epr-sequence (see Observation 2.9), we may assume, without loss of generality, that all of the diagonal entries of the leading $p \times p$ principal submatrix of $B$ are equal to 1. As each diagonal entry of $B$ is nonzero, the pigeonhole principle implies that $p \geq\left\lceil\frac{n}{2}\right\rceil$. Thus, it suffices to show that, for all integers $j$ with $3 \leq j \leq p, \ell_{j} \neq \mathrm{N}$. As $[\operatorname{epr}(B)]_{2}=\mathrm{A}, B[[p]]=I_{p}$ (otherwise, $B[[p]]$ would have a zero principal minor of order 2). Thus, for all integers $j$ with $3 \leq j \leq p, B[[j]]=I_{j}$ (which is nonsingular), implying that $\ell_{j} \neq \mathrm{N}$.

A corollary to Proposition 4.4 is that a symmetric matrix over $\mathbb{F}_{3}$ whose epr-sequence starts with AAN is a matrix of order at most 4:

Corollary 4.5. Let $B \in \mathbb{F}_{3}^{n \times n}$ be symmetric. Suppose that $\operatorname{epr}(B)=\mathrm{AAN}_{4} \ell_{5} \cdots \ell_{n}$. Then $n \leq 4$ and $\operatorname{epr}(B)$ is one of the following sequences:

$$
\text { AAN, AANA or AANN. }
$$

Proof. As $[\operatorname{epr}(B)]_{3}=$ N, Proposition 4.4 implies that $3>\left\lceil\frac{n}{2}\right\rceil$. It follows, then, that $n \leq 4$, as desired. Then, as $B$ is of order $n \geq 3$, and because $\ell_{n} \in\{\mathrm{A}, \mathrm{N}\}$, either $\operatorname{epr}(B)=$ AAN or $\operatorname{epr}(B)=$ AANA or epr $(B)=$ AANN, as desired.

We need the following lemma.

Lemma 4.6. Let $B \in \mathbb{F}_{3}^{n \times n}$ be symmetric. Suppose that $\operatorname{epr}(B)=\mathrm{AAA}_{4} \ell_{5} \cdots \ell_{n}$ and $B \neq I_{n}$ and $B \neq 2 I_{n}$. Then there exists a constant $c \in \mathbb{F}$ and a permutation matrix $P$ such that

$$
c P^{\top} B P=\left(\begin{array}{c|c}
I_{p} & F \\
\hline F^{\top} & 2 I_{n-p}
\end{array}\right),
$$

where $\left\lceil\frac{n}{2}\right\rceil \leq p \leq n-1$ and $F$ is a matrix with at most one nonzero entry in each row and each column. 
Proof. Suppose that $\operatorname{epr}(B)=\ell_{1} \ell_{2} \cdots \ell_{n}$. Thus, $\ell_{1} \ell_{2} \ell_{3}=$ AAA. As the square of any nonzero number in $\mathbb{F}_{3}$ is 1 , there is a constant $c \in \mathbb{F}$ such that the number of diagonal entries of $c B$ that are equal to 1 is larger than or equal to the number of diagonal entries that are equal to 2. Let $p$ be the number of diagonal entries of $c B$ that are equal to 1 . As multiplying a matrix by a nonzero constant leaves its epr-sequence invariant (see Observation 2.9), $\operatorname{epr}(c B)=\operatorname{epr}(B)$. Then, as $\ell_{1}=\mathrm{A}$, the pigeonhole principle implies that $p \geq\left\lceil\frac{n}{2}\right\rceil$. Then, as $n \geq 3, p \geq 2$. Let $P$ be a permutation matrix such that the first $p$ diagonal entries of $P^{\top} c B P$ are equal to 1 . Let $M=P^{\top} c B P$. By Observation 2.9, $\operatorname{epr}(M)=\operatorname{epr}(c B)=\operatorname{epr}(B)$. If $p=n$, then the fact that $\ell_{2}=$ A implies that $M=I_{n}$, which is a contradiction (because $B \neq I_{n}$ and $B \neq 2 I_{n}$ ). Thus, $\left\lceil\frac{n}{2}\right\rceil \leq p \leq n-1$. As $\ell_{1}=A$, each of the last $n-p$ diagonal entries of $M$ is equal to 2 . Then, as $\ell_{2}=\mathrm{A}$,

$$
M=\left(\begin{array}{c|c}
I_{p} & F \\
\hline F^{\top} & 2 I_{n-p}
\end{array}\right),
$$

for some matrix $F$. Note that $F=M[[p],[n] \backslash[p]]$. Let $M=\left[m_{i j}\right]$. Observe that, for $r, s \in[p]$ with $r \neq s$ and $t \in[n] \backslash[p] \operatorname{det}(M[r, s, t])=2-m_{r t}^{2}-m_{s t}^{2}$; then, as $\ell_{3}=\mathrm{A}$, either $m_{r t}$ or $m_{s t}$ is zero. Thus, each column of $F$ contains at most one nonzero entry. If $F$ has only one column, then the desired conclusion follows. Thus, we assume that $F$ has at least two columns, implying that $n-p \geq 2$. Now observe that, for $r \in[p]$ and $s, t \in[n] \backslash[p]$ with $s \neq t$, $\operatorname{det}(M[r, s, t])=2\left(2-m_{r s}^{2}-m_{r t}^{2}\right)$; then, as $\ell_{3}=$ A, either $m_{r s}$ or $m_{r t}$ is zero. Thus, $F$ contains at most one nonzero entry in each row.

With Lemma 4.6 at our disposal, we are ready to demonstrate that, if a given epr-sequence over $\mathbb{F}_{3}$ starts with $\mathrm{AAA}$, then the sequence is AAAA .

Theorem 4.7. Let $B \in \mathbb{F}_{3}^{n \times n}$ be symmetric. Suppose that $\operatorname{epr}(B)=\mathrm{AAA}_{4} \ell_{5} \cdots \ell_{n}$. Then $\operatorname{epr}(B)=\mathrm{AAA} \overline{\mathrm{A}}$.

Proof. As the desired conclusion follows immediately if $B=I_{n}$ or $B=2 I_{n}$, assume that $B \neq I_{n}$ and $B \neq 2 I_{n}$. Because of Lemma 4.6, and because any two permutationally similar matrices have the same epr-sequence, and because the epr-sequence of a matrix is preserved after multiplication by a nonzero constant (see Observation 2.9), we may assume, without loss of generality, that

$$
B=\left(\begin{array}{c|c}
I_{p} & F \\
\hline F^{\top} & 2 I_{n-p}
\end{array}\right),
$$

where $\left\lceil\frac{n}{2}\right\rceil \leq p \leq n-1$ and $F$ is a matrix with at most one nonzero entry in each row and each column.

As there is nothing to prove if $n=3$, we assume that $n \geq 4$. Let $k$ be an integer with $4 \leq k \leq n$ and $C$ be a $k \times k$ principal submatrix of $B$. It suffices to show that $C$ is nonsingular. Observe that either $C=I_{k}$ or $C=2 I_{k}$ or

$$
C=\left(\begin{array}{c|c}
I_{q} & G \\
\hline G^{\top} & 2 I_{k-q}
\end{array}\right),
$$

where $1 \leq q \leq k-1$ and $G$ is a matrix with at most one nonzero entry in each row and each column. Observe that $G^{\top} G$ is a diagonal matrix all of whose diagonal entries are either 0 or 1 . As $C[[q]]=I_{q}$ is nonsingular, the Schur Complement Theorem implies that $\operatorname{det}(C)=\operatorname{det}(C[[q]]) \operatorname{det}(C / C[[q]])$, where $C / C[[q]]$ is the Schur complement of $C[[q]]$ in $C$. Thus, $\operatorname{det}(C)=\operatorname{det}(C / C[[q]])$, implying that it suffices to show that $C / C[[q]]$ is nonsingular. Observe that

$$
C / C[[q]]=2 I_{k-q}-G^{\top}(C[[q]])^{-1} G=2 I_{k-q}-G^{\top}\left(I_{q}\right)^{-1} G=2 I_{k-q}-G^{\top} G,
$$

which is a diagonal matrix all of whose diagonal entries are either 1 or 2, implying that $C / C[[q]]$ is nonsingular, as desired.

We need another lemma. 
Lemma 4.8. Let $B \in \mathbb{F}_{3}^{n \times n}$ be symmetric. Suppose that $\operatorname{epr}(B)=\mathrm{NA}_{3} \ell_{4} \cdots \ell_{n}$. If $\ell_{4}=\mathrm{A}$, then $n \leq 6$ and $\operatorname{epr}(B)$ is one of the following sequences:

$$
\text { NAAA, NAAAN, NAAANA. }
$$

Proof. Suppose that $\ell_{4}=\mathrm{A}$ and $\operatorname{epr}(B)=\ell_{1} \ell_{2} \cdots \ell_{n}$. Thus, $\ell_{1} \ell_{2}=$ NA. By Corollary $3.8, n \leq 6$. As NAN and NAS are forbidden (by Theorem 2.6), $\ell_{3}=$ A. If $n=4$, then $\operatorname{epr}(B)=$ NAAA, as desired. Assume that $n \geq 5$. If $\ell_{5} \in\{\mathrm{A}, \mathrm{S}\}$, then, by the Inheritance Theorem, $B$ has a $5 \times 5$ principal submatrix $C$ with $\operatorname{epr}(C)=$ NAAAA, which implies that epr $\left(C^{-1}\right)=$ AAANA (see the Inverse Theorem), contradicting Theorem 4.7. It follows, then, that $\ell_{5}=$ N. If $n=5$, then $\operatorname{epr}(B)=$ NAAAN, as desired. Assume that $n=6$.

We have already shown that $\operatorname{epr}(B)=\mathrm{NAAAN}_{6}$. Thus, it remains to show that $\ell_{6}=\mathrm{A}$; that is, it remains to show that $B$ is nonsingular. As $\ell_{1} \ell_{2}=N A$, each off-diagonal entry of $B$ is nonzero. Without loss of generality, we may assume that each off-diagonal entry in the first row and first column of $B$ is equal to 1 (see Observation 2.10). Let $M$ be the $5 \times 5$ matrix obtained from $B[\{2,3,4,5,6\}]$ by replacing any off-diagonal entries that are equal to 2 with 0 . Let $G$ be the graph such that $A_{\mathbb{F}_{3}}(G)=M$ (as each diagonal entry of $B$ is zero, such a graph exists). We will now show that both $G$ and $\bar{G}$ are triangle-free; it suffices to show that neither $J_{3}-I_{3}$ nor $2\left(J_{3}-I_{3}\right)$ is a principal submatrix of $B[\{2,3,4,5,6\}]$. If $B[\{i, j, k\}]=J_{3}-I_{3}$ or $B[\{i, j, k\}]=2\left(J_{3}-I_{3}\right)$ for some $i, j, k \in\{2,3,4,5,6\}$, then $B[\{1, i, j, k\}]$ is singular, which contradicts the fact that $\ell_{4}=A$. Thus, both $G$ and $\bar{G}$ are triangle-free. As $G$ is of order 5, Theorem 2.8 implies that $G=C_{5}$. Thus, $B[\{2,3,4,5,6\}]$ is permutationally similar to $A_{\mathbb{F}_{3}}\left(C_{5}\right)+2 A_{\mathbb{F}_{3}}\left(\overline{C_{5}}\right)$. Then, as each entry in the first row and first column of $B$ is equal to 1 , and because the first diagonal entry of $B$ is zero, $B$ is uniquely determined, up to permutation similarity; moreover, $B$ is permutationally similar to the nonsingular matrix in Example 3.9. It follows that $B$ is nonsingular, as desired.

A symmetric matrix over $\mathbb{F}_{3}$ whose epr-sequence contains NAAA as a subsequence is a matrix of order at most 6:

Proposition 4.9. If NAAA occurs as a subsequence of the epr-sequence of a symmetric matrix $B$ over $\mathbb{F}_{3}$, then $n \leq 6$ and $\operatorname{epr}(B)$ is one of the following sequences:

$$
\text { NAAA, NAAAN, NAAANA. }
$$

Proof. Let $B$ be an $n \times n$ symmetric matrix over $\mathbb{F}_{3}$, and let epr $(B)=\ell_{1} \ell_{2} \cdots \ell_{n}$. Suppose that $\ell_{k} \ell_{k+1} \ell_{k+2} \ell_{k+3}=$ NAAA for some $k \in[n-3]$. Because of Lemma 4.8, it suffices to show that $k=1$. Let $B^{\prime}$ be a $(k+3) \times(k+3)$ principal submatrix of $B$, and let $\operatorname{epr}\left(B^{\prime}\right)=\ell_{1}^{\prime} \ell_{2}^{\prime} \cdots \ell_{k+3}^{\prime}$. By the Inheritance Theorem, epr $\left(B^{\prime}\right)=\ell_{1}^{\prime} \ell_{2}^{\prime} \cdots \ell_{k-1}^{\prime}$ NAAA. By the Inverse Theorem, $\operatorname{epr}\left(\left(B^{\prime}\right)^{-1}\right)=\mathrm{AAN}_{k_{-1}}^{\prime} \cdots \ell_{2}^{\prime} \ell_{1}^{\prime} \mathrm{A}$. It follows from Corollary 4.5 that $\left(B^{\prime}\right)^{-1}$ is of order at most 4. Thus, $k+3 \leq 4$, implying that $k=1$.

If the epr-sequence of a given symmetric matrix in $\mathbb{F}_{3}^{n \times n}$ starts with NAAN, then the sequence is completely determined by the value of $n$ :

Proposition 4.10. Let $B \in \mathbb{F}_{3}^{n \times n}$ be symmetric. Suppose that $\operatorname{epr}(B)=\mathrm{NAAN}_{5} \ell_{6} \cdots \ell_{n}$. Then epr $(B)$ is of one of the following forms:

$$
\text { NAAN } \overline{A A N}, \quad \text { NAAN } \overline{A A N} A, \quad \text { NAAN } \overline{A A N} A \text {. }
$$

Proof. Because of Proposition 4.1, it suffices to show that epr $(B)=\operatorname{epr}\left(J_{n}-I_{n}\right)$. As $[\operatorname{epr}(B)]_{1}^{2}=$ NA, each offdiagonal entry of $B$ is nonzero. Because of Observation 2.10, we may assume that each off-diagonal entry in the first row and first column of $B$ is equal to 1 . We claim that either $B[\{2,3, \ldots, n\}]=J_{n-1}-I_{n-1}$ or $B[\{2,3, \ldots, n\}]=2\left(U_{n-1}-I_{n-1}\right)$. Suppose that this claim is false. As each off-diagonal entry of $B$ is nonzero, and because each diagonal entry of $B$ is zero, and because $B$ is symmetric, $B[\{2,3, \ldots, n\}]$ has a row containing both a 1 and a 2 as off-diagonal entries. Suppose that $B=\left[b_{i j}\right]$. As any two permutationally similar matrices have the same epr-sequence (see Observation 2.9), we may assume, without loss of generality, that $b_{23}=1$ and $b_{24}=2$. Observe that $\operatorname{det}(B[\{1,2,3,4\}])=1+\left(b_{34}\right)^{2}$. As $b_{34} \neq 0,\left(b_{34}\right)^{2}=1$, implying that 
$\operatorname{det}(B[\{1,2,3,4\}]) \neq 0$, which contradicts the fact that $[\operatorname{epr}(B)]_{4}=\mathrm{N}$. Thus, our claim is true. We proceed by considering the two possible cases based on $B[\{2,3, \ldots, n\}]$.

Case 1: $B[\{2,3, \ldots, n\}]=J_{n-1}-I_{n-1}$.

As each off-diagonal entry in the first row and first column of $B$ is equal to 1 , and because $b_{11}=0, B=J_{n}-I_{n}$, implying that $\operatorname{epr}(B)=\operatorname{epr}\left(J_{n}-I_{n}\right)$, as desired.

Case 2: $B[\{2,3, \ldots, n\}]=2\left(J_{n-1}-I_{n-1}\right)$.

Let $M$ be the matrix that results from multiplying the first row and first column of $B$ by 2 . Then, as multiplication of any row or column of a matrix by a nonzero constant leaves the rank of every submatrix invariant, $\operatorname{epr}(B)=\operatorname{epr}(M)$. As $M=2\left(J_{n}-I_{n}\right)$, and because $\operatorname{epr}(M)=\operatorname{epr}\left(J_{n}-I_{n}\right)$ (see Observation 2.9), $\operatorname{epr}(B)=\operatorname{epr}\left(U_{n}-I_{n}\right)$, as desired.

The next example exhibits two matrices of which we shall make use in the proof of Theorem 4.12.

Example 4.11. For the following symmetric matrices over $\mathbb{F}_{3}$, epr $\left(M_{\sigma}\right)=\sigma$ :

$$
M_{\mathrm{AANA}}=\left(\begin{array}{cccc}
1 & 0 & 1 & 1 \\
0 & 1 & 1 & 1 \\
1 & 1 & 2 & 0 \\
1 & 1 & 0 & 2
\end{array}\right) \quad \text { and } \quad M_{\mathrm{AANN}}=\left(\begin{array}{cccc}
1 & 0 & 1 & 1 \\
0 & 1 & 1 & 2 \\
1 & 1 & 2 & 0 \\
1 & 2 & 0 & 2
\end{array}\right) .
$$

We are finally ready to establish the main result of this section.

Theorem 4.12. Let $n \geq 3$ and $\sigma=\ell_{1} \ell_{2} \cdots \ell_{n}$ be a sequence from $\{\mathrm{A}, \mathrm{N}\}$. Then there exists a symmetric matrix in $\mathbb{F}_{3}^{n \times n}$ whose epr-sequence is $\sigma$ if and only if $\sigma$ is of one of the following forms:
1. $A A A \bar{A}$.
5. ANA $\overline{A N A}$.
9. NAA.
13. NAAN $\overline{A A N}$.
2. AAN.

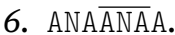
10. NAAA.
14. NAAN $\overline{A A N} A$.
3. AANA.
7. ANA $\overline{A N A} A N$.
11. NAAAN.
15. NAAN $\overline{A A N} A A$.
4. AANN.
8. ANN $\bar{N}$.
12. NAAANA.

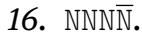

Proof. Suppose that there exists a symmetric matrix $B \in \mathbb{F}_{3}^{n \times n}$ with $\operatorname{epr}(B)=\sigma$. By the NN Theorem, $\ell_{1} \ell_{2} \ell_{3} \neq$ NNA. Then, as NAN is forbidden, $\ell_{1} \ell_{2} \ell_{3} \in\{$ AAA, AAN, ANA, ANN, NAA, NNN $\}$. If $\ell_{1} \ell_{2} \ell_{3}=$ AAA, then $\sigma=$ AAAA (see Theorem 4.7). If $\ell_{1} \ell_{2} \ell_{3}=$ AAN, Corollary 4.5 implies that either $\sigma=$ AAN or $\sigma=$ AANA or $\sigma=$ AANN. If $\ell_{1} \ell_{2} \ell_{3}=$ ANA,

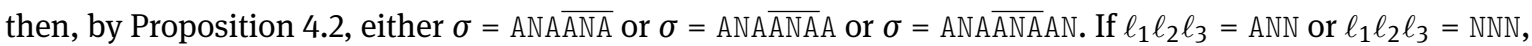
then the NN Theorem implies that either $\sigma=\operatorname{AnNN\overline {N}}$ or $\sigma=$ NNNN $\bar{N}$.

Finally, we consider the remaining case: $\ell_{1} \ell_{2} \ell_{3}=$ NAA. If $n=3$, then $\sigma=$ NAA. Suppose that $n \geq 4$. We proceed by considering two cases, based on $\ell_{4}$.

Case 1: $\ell_{4}=\mathrm{A}$.

It follows that $\sigma=\operatorname{NAAA}_{5} \ell_{6} \cdots \ell_{n}$ and, therefore, by Proposition 4.9, either $\sigma=$ NAAA or $\sigma=$ NAAAN or $\sigma=$ NAAANA.

Case 2: $\ell_{4}=\mathrm{N}$.

It follows from Proposition 4.10 that either $\sigma=$ NAAN $\overline{A A N}$ or $\sigma=$ NAAN $\overline{A A N A}$ or $\sigma=$ NAAN $\overline{A A N A A}$.

We will now establish the other direction. If $\sigma$ is of the form AAAA $\bar{A}$, then $\sigma=\operatorname{epr}\left(I_{n}\right)$. If $\sigma=$ AAN, then, by the Inheritance Theorem, $\sigma$ is the epr-sequence of any of the $3 \times 3$ principal submatrices of the matrix $M_{\text {AANA }}$ in Example 4.11. If $\sigma=$ AANA or $\sigma=$ AANN, then $\sigma$ is the epr-sequence of one of the matrices in Example 4.11. If $\sigma$ is of one of the following forms, then $\sigma=\operatorname{epr}\left(U_{n}-2 I_{n}\right)$ : ANA $\overline{A N A}$, ANAANAA, and ANA $\overline{A N A} A N$ (see Proposition 4.1). If $\sigma$ is of the form ANN $\bar{N}$, then $\sigma=\operatorname{epr}\left(J_{n}\right)$. If $\sigma=\mathrm{NAA}$, then $\sigma=\operatorname{epr}\left(J_{3}-I_{3}\right)$. If $\sigma=\mathrm{NAAA}$, then $\sigma=\operatorname{epr}\left(\left(M_{\text {AANA }}\right)^{-1}\right)$ (see the Inverse Theorem). If $\sigma=$ NAAAN, then, by the Inheritance Theorem, $\sigma$ is the epr-sequence of any of the $5 \times 5$ principal submatrices of the matrix $M_{\text {NAAANA }}$ in Example 3.9; and, if $\sigma=$ NAAANA, then $\sigma=\operatorname{epr}\left(M_{\text {NAAANA }}\right)$. If $\sigma$ is of one of the following forms, then $\sigma=\operatorname{epr}\left(J_{n}-I_{n}\right)$ : NAAN $\overline{A A N}$, NAAN $\overline{A A N A}$ and NAAN $\overline{A A N A A}$ (see Proposition 4.1). If $\sigma$ is of the form NNNN, then $\sigma=\operatorname{epr}\left(O_{n}\right)$. 
Acknowledgments: The research of Peter Dukes is supported by the NSERC grant RGPIN-312595-2017. The research of Xavier Martínez-Rivera was supported by the following NSERC grants: RGPIN-3677-2016 and RGPIN-312595-2017.

Data Availability Statement: Data sharing is not applicable to this article as no datasets were generated or analyzed during the current study.

\section{References}

[1] W. Barrett, S. Butler, M. Catral, S. M. Fallat, H. T. Hall, L. Hogben, P. van den Driessche, M. Young. The principal rank characteristic sequence over various fields. Linear Algebra and its Applications 459 (2014), 222-236.

[2] R. A. Brualdi, L. Deaett, D. D. Olesky, P. van den Driessche. The principal rank characteristic sequence of a real symmetric matrix. Linear Algebra and its Applications 436 (2012), 2137-2155.

[3] R. A. Brualdi, H. Schneider. Determinantal identities: Gauss, Schur, Cauchy, Sylvester, Kronecker, Jacobi, Binet, Laplace, Muir, and Cayley. Linear Algebra and its Applications 52/53 (1983), 769-791.

[4] S. Butler, M. Catral, S. M. Fallat, H. T. Hall, L. Hogben, P. van den Driessche, M. Young. The enhanced principal rank characteristic sequence. Linear Algebra and its Applications 498 (2016), 181-200.

[5] R. L. Graham, B. L. Rothschild, J. H. Spencer. Ramsey theory. Wiley Series in Discrete Mathematics and Optimization. John Wiley \& Sons, Inc., Hoboken, NJ, 2013.

[6] O. Holtz, H. Schneider. Open problems on GKK $\tau$-matrices. Linear Algebra and its Applications 345 (2002), 263-267.

[7] X. Martínez-Rivera. Classification of families of pr- and epr-sequences. Linear and Multilinear Algebra 65 (2017), $1581-1599$.

[8] X. Martínez-Rivera. The enhanced principal rank characteristic sequence over a field of characteristic 2. Electronic Journal of Linear Algebra 32 (2017), 273-290.

[9] S. Radziszowski. Small Ramsey numbers. Electronic Journal of Combinatorics DS1, 2017.

[10] F. Zhang (editor). The Schur Complement and its Applications. Springer-Verlag, New York, New York, 2005. 